\title{
Danger de confusion entre Prava et les préparations à base de pravastatine
}

Swissmedic

Correspondance:

Swissmedic

Hallerstrasse 7

Case postale

CH-3000 Berne 9

www.swissmedic.ch
Concerne Prava, capsules (lomustine, cytostatique) et les génériques/médicaments en comarketing à base de pravastatine (inhibiteurs de la HMG-CoA réductase; statines) suivants:

- Pravastatine Nycomed, comprimés;

- Pravalotin, comprimés;

- Pravastatine Sandoz, comprimés;

- Pravatine, comprimés,

- Pravasta eco, comprimés;

- Pravastatine Helvepharm, comprimés;

- Pravastatine Streuli, comprimés;

- Pravastatin-Teva, comprimés;

- Pravastax, comprimés.

Swissmedic a été informé de confusions ou de risques de confusion lors de la prescription de préparations à base de pravastatine (inhibiteurs de la HMG-CoA réductase à effet hypocholestérolémiant) avec le cytostatique Prava (lomustine).

C'est pourquoi le titulaire de l'autorisation de Prava, Bristol-Myers Squibb, se propose de modifier le nom de sa préparation et a d'ores et déjà pris les mesures qui s'imposent. Toutefois, il reste des emballages portant le nom Prava dans le commerce.
Swissmedic demande à tous les médecins de formuler les noms de préparation et les recommandations posologiques des préparations à base de pravastatine en entier et avec clarté sur les ordonnances.

Dans le même temps, tous les pharmaciens se voient priés de vérifier chaque ordonnance de Prava et de s'assurer qu'il s'agit bien du cytostatique.

Les recommandations posologiques usuelles du cytostatique Prava et celles des préparations à base de pravastatine se distinguent considérablement (Prava: 100-130 mg/m² de surface corporelle en dose unique toutes les 6-8 semaines; préparations à base de pravastatine: $10-40 \mathrm{mg}$ 1 fois par jour).

Swissmedic enjoint les médecins et les pharmaciens d'appliquer systématiquement les mesures de précaution susmentionnées (vérification minutieuse de l'indication et de la posologie) pour chaque prescription de médicament. En outre, les patients doivent toujours être encouragés à lire la notice d'emballage. 